\title{
HOMOTOPICAL NILPOTENCE OF THE SEVEN SPHERE
}

\author{
WILLIAM J. GILBERT ${ }^{1}$
}

Abstract. We prove that the homotopical nilpotence of $S^{7}$ is 3 , with respect to any of its $120 \mathrm{H}$-space multiplications.

The homotopical nilpotence of $S^{3}$ has been calculated by Porter [4] for the standard multiplication and by Arkowitz and Curjel [1] for all of its twelve $H$-space multiplications. Arkowitz and Curjel mention that their methods lead to results on the multiplications on $S^{7}$ but do not calculate its homotopical nilpotence. By modifying their arguments with the Samelson products we obtain the results on $S^{7}$ easily.

We will denote the collection of homotopy classes of basepoint preserving maps from $A$ to $B$ by $[A, B]$ and we will not distinguish notationally between a map and its homotopy class. The multiplication and inverse in the unit Cayley numbers induce the standard multiplication $m \in\left[S^{7} \times S^{7}\right.$, $\left.S^{7}\right]$ and two sided homotopy inverse $v \in\left[S^{7}, S^{7}\right]$ on the space $S^{7}$. For the $H$-space $\left(S^{7}, m, v\right)$ we define a commutator map $\phi: S^{7} \times S^{7} \rightarrow S^{7}$ by $\phi(x, y)=$ $(x \cdot y) \cdot\left(x^{-1} \cdot y^{-1}\right)$ using the multiplication $m$ and inverse $v$. Recall that the Cayley multiplication is not associative but is diassociative, i.e. any two elements generate an associative subalgebra. We now make a choice in bracketing to define inductively the $k$-fold commutator map $\phi:\left(S^{7}\right)^{k} \rightarrow S^{7}$ by $\phi_{k}=\phi \circ\left(\phi_{k-1} \times 1\right)$ where $\phi_{1}=1$, the identity map on $S^{7}$. It is well known that $\phi_{k}$ induces a unique homotopy class $\psi_{k} \in\left[\bigwedge^{k} S^{7}, S^{7}\right]$ with $\psi_{k} \circ q_{k}=\phi_{k}$, where $\Lambda^{k} S^{7}$ is the $k$-fold smash product of $S^{7}$ (homeomorphic to $S^{7 k}$ ) and $q_{k}:\left(S^{7}\right)^{k} \rightarrow \Lambda^{k} S^{7}$ is the projection map. The homotopical nilpotence of the $H$-space $\left(S^{7}, m, v\right)$ written $\operatorname{nil}\left(S^{7}, m, v\right)$, is the least integer $k$ such that $\phi_{k+1}$ (and hence $\psi_{k+1}$ ) is nullhomotopic.

TheOREM. $\operatorname{nil}\left(S^{7}, m, v\right)=3$.

Proof. James [2, p.176] proves that $\psi_{2}$ generates $\pi_{14}\left(S^{7}\right)=Z_{120}$ so that in Toda's notation [5] its 2-component is $\sigma^{\prime}$, its 3-component is $\alpha_{2}$ (7) and its 5-component is $\alpha_{1}(7)$. Now

$$
\psi_{3}=\psi \circ(\psi \wedge 1)=\psi \circ \Sigma^{7} \psi \in \pi_{21}\left(S^{7}\right)=Z_{24} \oplus Z_{4}
$$

Received by the editors June 21, 1971.

AMS 1970 subject classifications. Primary 55D45.

Key words and phrases. Homotopical nilpotence, $H$-space, commutator map.

${ }^{1}$ Supported in part by a National Research Council grant. 
and its 2-component is $\sigma^{\prime} \circ \Sigma^{7} \sigma^{\prime}=2 \sigma^{\prime} \circ \sigma_{14} \neq 0$ [5]. The element $\alpha_{2}$ is defined in terms of a Toda bracket and so the 3-component of $\psi_{3}$ is

$$
\begin{aligned}
\alpha_{2}(7) \circ \alpha_{2}(14) & \in\left\{\alpha_{1}(7), 3 \iota_{10}, \alpha_{1}(10)\right\} \circ \alpha_{2}(14) \\
\subset & \left\{\alpha_{1}(7), 3 \iota_{10}, \alpha_{1}(10) \circ \alpha_{2}(13)\right\}=0
\end{aligned}
$$

since $\alpha_{1}(10) \circ \alpha_{2}(13)=0$ by Lemma 13.8 of [5]. Hence $\psi_{3}$ has only a 2component and

$$
\psi_{4}=\psi_{3} \circ \Sigma^{14} \psi \in \pi_{28}\left(S^{7}\right)=Z_{6} \oplus Z_{2} \text { by [3] }
$$

and so $\psi_{4}=4 \sigma^{\prime} \circ \sigma_{14} \circ \sigma_{21}=0$ which proves the theorem.

There are 120 different homotopy classes of multiplications on $S^{7}$ and as in Lemma 2 of [1] it can be shown that they can be written additively in the form

$$
m^{(t)}=m+t \phi \in\left[S^{7} \times S^{7}, S^{7}\right], \quad t=0,1, \cdots, 119 .
$$

Also as in Lemma 3 of [1], $v$ is a homotopy inverse for each of these multiplications.

COROllary. $\quad \operatorname{nil}\left(S^{7}, m^{(t)}, v\right)=3$ for $t=0,1, \cdots, 119$.

Proof. Denote by $\psi_{k}^{(t)} \in\left[\bigwedge^{k} S^{7}, S^{i}\right]$ the $r$-fold smash commutator map defined on the $H$-space $\left(S^{7}, m^{(t)}, v\right)$. Then James [2, p. 176] and Arkowitz and Curjel [1, Lemma 4] prove that $\psi_{k}^{(t)}=(2 t+1) \psi_{k}$. Hence $\psi_{3}^{(t)}$ is nonzero and $\psi_{4}^{(t)}$ is zero, which proves the corollary.

Changing the choice of bracketing in the definition of the $k$-fold commutator map will at most affect a sign change in $\psi_{k}$, so that the homotopy nilpotence is independent of the choice of bracketing.

\section{REFERENCES}

1. M. Arkowitz and C. R. Curjel, Some properties of the exotic multiplications on the three-sphere, Quart. J. Math. Oxford Ser. (2) 20 (1969), 171-176. MR 40 \#2066.

2. I. M. James, On H-spaces and their homotopy groups, Quart. J. Math. Oxford Ser. (2) 11 (1960), 161-179. MR 24 \#A2966.

3. M. Mimura, On the generalized Hopf homomorphism and the higher composition. II, $\pi_{n+i}\left(S^{n}\right)$ for $i=21$ and 22, J. Math. Kyoto Univ. 4 (1965), 301-326. MR 31 \#1676.

4. G. J. Porter, Homotopical nilpotence of $S^{3}$, Proc. Amer. Math. Soc. 15 (1964), 681-682. MR 29 \#6496.

5. H. Toda, Composition methods in homotopy groups of spheres, Ann. of Math. Studies, no. 49, Princeton Univ. Press, Princeton, N.J., 1962. MR 26 \#777.

Department of Pure Mathematics, University of Waterloo, Waterloo, Ontario, Canada 